\title{
Chronic biological false-positive reactions to serological tests for syphilis in blood donors
}

\author{
M. F. GARNER AND J. L. BACKHOUSE
}

From the Institute of Clinical Pathology and Medical Research, Lidcombe, Sydney, Australia

SYNOPSIS Problem sera from 375 blood donors were investigated for biological falses positive reactions to serological tests for syphilis. Problem sera are those sera in which su震 mitting laboratories have found a reactive result to a screening test for syphilis. On each serum a cardiolipin Wassermann reaction, a Venereal Disease Research Laboratory test, a Reitär protein-complement fixation test, a Treponema pallidum immobilization test, and a fluoresceng treponemal antibody absorption test were performed.

Of the sera $49.9 \%$ were found to be non-reactive in all five tests, $28.8 \%$ gave results indicating a diagnosis of syphilis, and $21.3 \%$ showed biological false-positive reactions.

There were 80 sera from blood donors which gave biological false-positive reactions. further specimen of serum from 67 of these donors was tested after an interval of a minimuğ of six months. Sixty-six of the sera showed chronic biological false-positive reactions. Some donors were only found reactive for the first time after they had given very many donation of blood.

It is concluded that sudden blood loss, as in blood donation, appears to stimulate the production of excess reagin in certain individuals, causing a chronic biological false positive reaction to serological tests for syphilis. This may only appear after several blood donations have been made.

The introduction of the Treponema pallidum immobilization (TPI) and fluorescent treponemal antibody absorption tests, both specific tests for syphilis, has helped to clarify the problem of nonspecific reactive results to routine serologial tests for syphilis. These non-specific reactions, usually called biological false-positive reactions, are, in the majority of cases, reactive results to reagin detection tests in the absence of any history or clinical signs of infection. There are two types of biological false-positive reactions: acute, which tend to disappear in a few days to a few months, and chronic, which last over six months and sometimes for life.

In a recent review of 753 problem sera sent to the laboratory for TPI testing it was found that of 88 sera from blood donors, 22 gave biological

Received for publication 19 January 1970. false-positive reactions and a further 22 reactio indicating syphilis (Garner, 1970). It was felt that blood loss may, in some individuals, responsible for the production of excess reagin and false-positive reactions to serological tes ts $^{2}$ for syphilis.

The study reported here was undertaken to see if blood donors whose sera were showing bie logical false-positive reactions at the time of donation were giving acute or chronic reactions.

\section{Materials and Methods}

The sera received from blood donors were aी 'problem' sera, that is, a reactive screening test for syphilis had been found by the submitting laboratory. The following tests were carried out 
on each serum: cardiolipin Wassermann reaction (CWR), Venereal Disease Research Laboratory (VDRL) test, Reiter protein-complement fixation (RPCF) test, fluorescent treponemal antibody absorption (FTA-ABS) test, and a Treponema pallidum immobilization (TPI) test.

Those sera which gave non-reactive FTA-ABS and TPI test results and reactive results in any of the other three tests were considered to give non-specific or biological false-positive reactions. Another specimen of serum from these donors was tested after a minimum of six months from the finding of the original biological false-positive reaction.

\section{Results}

Problem sera were received from 375 blood donors. One hundred and eighty-seven or $49.9 \%$ of the sera were found to be non-reactive in all five tests and therefore these sera were from donors who neither had syphilis nor did their sera show biological false-positive reactions. The reactive screening test results found on these sera by the submitting laboratories were probably due to the use of oversensitive antigens or faulty technique. One hundred and eight or $28.8 \%$ of the sera gave reactive results to some or all of the CWR, VDRL, and RPCF tests, as well as reactive TPI and FTA-ABS test results. Thus these 108 sera were from donors who either had or had had syphilis. The remaining 80 or $21 \cdot 3 \%$ of the sera showed reactive results to the reagin and or RPCF tests and non-reactive TPI and FTA-ABS test results, ie, showed biological false-positive reactions (Table).

\begin{tabular}{lc}
\hline Result of Reaction & No. of Sera \\
\hline Neither syphilis nor biological false-positive & \\
$\quad$ reaction & $187(49 \cdot 9 \%)$ \\
Syphilis & $108(28 \cdot 8 \%)$ \\
Biological false-positive & $80(21 \cdot 3 \%)$ \\
Total & $375(100 \%)$ \\
\hline
\end{tabular}

Table Results on serological testing of 375 problem sera from blood donors

Another specimen of serum was tested from 67 of these 80 blood donors after an interval of from six months to two years. The remaining 13 blood donors in the group either could not be contacted or refused to cooperate.

Serum from one donor which had shown a weakly reactive VDRL test result on initial testing was non-reactive in all five tests when the second specimen was examined. This was the only serum to show an acute biological falsepositive reaction.

Sera from the remaining 66 blood donors gave non-reactive TPI and FTA-ABS test results and similar reactive results to the CWR, VDRL, $\frac{\varrho}{\overline{5}}$ and/or RPCF tests, as was found when the original 0 specimen of serum was tested. These 66 sera, $\stackrel{0}{\vec{*}}$ which showed chronic biological false-positive 을 reactions, gave reactive test results as follows: $\overrightarrow{\vec{F}}$ CWR only 3, VDRL only $29, \mathrm{CWR}$ and VDRL only 28 , VDRL and RPCF only 1, and CWR, VDRL, and RPCF only 5.

\section{Discussion}

This paper deals only with problem sera from $\overrightarrow{\vec{\omega}}$ blood donors and the significance of the per- ${ }^{\circ}$ centage of biological false-positive and syphilitic reactions found must be viewed in this context. in The blood donors in this survey were all appar- 0 ently healthy, unpaid volunteers who showed no obvious signs of illness.

The majority of biological false-positive reactions found were chronic, only one in $67-$ being an acute reaction. Of the 66 sera which $\$$ showed chronic false-positive reactions, 64 were $\frac{\mathbb{}}{\mathscr{P}}$ from people who had given many blood donations $\frac{\mathbb{}}{3}$ and two were found at the first donation. The submitting laboratory reported that a reactive screening test was first found in the serum of $32 \overrightarrow{0}$ donors as follows: four at the second, one at the $\odot$ third, three at each of the fourth, fifth, sixth, seventh and eighth, one at the tenth, three at the nineteenth, two at the twentieth, two at the twenty-second, and four at the twenty-fourth donation. Thus, multiple blood donation appears $\mathbb{D}$ to increase the possibility of certain donors becoming chronic false-positive reactors. Moore윽 and Mohr in 1952 listed repeated blood loss, as in multiple donations for transfusion, as a cause of biological false-positive reactions, but did not distinguish between acute and chronic reactions.

As would be expected in sera showing bio-i logical false positive reactions, the VDRL titre was higher than the corresponding CWR titre in most of the 28 sera in this group.

This investigation differs from that of Barnardo and Rein (1946), in that they found transient $>$ reactions following blood donation. They investi-을 gated 300 healthy seronegative blood donors. Postdonation sera were examined, biological ${ }^{\text {or }}$ false-positive reactions being shown by almost ${ }_{0}^{N}$ $10 \%$, most of which converted to non-reactive $\mathcal{E}^{N}$ within eight weeks following the original bloodo donation. They suggested that bleeding could be aco factor in producing positive serological reactions $\frac{\varnothing}{\varnothing}$ for syphilis. However, their series were of acute? biological false-positive reactions following blood 0 donation, whereas our series is of biologicalo false-positive reactions first found at the time of ${ }_{\rho}^{\mathbb{D}}$ blood donation, these reactions still being present $\frac{}{\Phi}$ at least six months after the original donation and thus chronic reactions.

It appears from our results that in susceptible individuals sudden blood loss, as in blood dona- 
tion, can stimulate the immune mechanism to produce excess reagin, with resultant biological false-positive reactions to serological tests for syphilis. Most of these seem to be chronic reactions. In certain people multiple blood donation plays some part in the production of the chronic reaction, probably due to repeated stimulation of the immune mechanism.

Reactive screening tests for syphilis found in the serum of blood donors should always be investigated further, preferably by using the TPI and FTA-ABS tests, as, apart from biologic false-positive reactions, a number will indicate diagnosis of syphilis.

\section{References}

Barnard, R. D., Rein, C. R., and Doan, C. A. (1946). FaBe positive serologic tests for syphilis following blood $\frac{\mathrm{O}-}{-}$ nation. Amer. J. Syph., 30, 255-263.

Garner, M. F. (1970). The biological false positive reaction $\frac{\omega}{D 0}$ serological tests for syphilis. J. clin. Path., 23, 31-34.

Moore, J. E., and Mohr, C. F. (1952). Biologically false positive serologic tests for syphilis. J. Amer. med. Ass., 150, 467-4 Z.

\section{Reports and Bulletins prepared by the Association of Clinical Biochemists}

The following reports and bulletins are published by the Association of Clinical Biochemists. They may be obtained from The Administrative Office, Association of Clinical Biochemists, 7 Warwick Court, Holborn, London, WC1R 5DP. The prices include postage, but airmail will be charged extra. Overseas readers should remit by British Postal or Money Order. If this is not possibte the equivalent of 10s. is the minimum amount that can be accepted.

\section{SCIENTIFIC REPORTS}

3 Automatic Dispensing Pipettes. An assessment of 35 commercial instruments 1967 P. M. G. BROUGHTON, A. H. GOWENLOCK, G. M. WIDDOWSON, and K. A. AHLQUIST 17s $(\$ 2)$

4 An Evaluation of 5 Commercial Flame Photometers suitable for the Simultaneous Determination of Sodium and Potassium March 1970 P. M. G. BROUGHTON and J. B. DAWSON 17s (\$2)

\section{TECHNICAL BULLETINS}

9 Determination of Urea by AutoAnalyzer November 1966 RUTH M. HASLAM 8s 6d (\$1)

10 Filter Fluorimeters. A comparative list of 14 instruments March 1967 HANNELORE BRAUNSBERG (Re-issued in response to demand. Text still valuable, list now out of date) $8 \mathrm{~s} 6 \mathrm{~d}(\$ 1)$
11 Determination of Serum Albumin by AutoAnalyzer using Bromocresol Green October 1967 B. E. NORTHAM and G. M. WIDDOWSON 8s 6d (\$1)

13 An Assessment of the Technicon Type II Sampler Unit March 1968 B. C. GRAY and G. K. MCGOWAN 8s 6d (\$1)

14 Atomic Absorption Spectroscopy. An outline of its principles and a guide to the selection of instruments May 1968 J. B. DAWSON and P. M. G. BROUGHTON 8s 6d (\$1)

15 A Guide to Automatic Pipettes (2nd edition) June 1968 P. M. G. BROUGHTON 8s $6 \mathrm{~d}(\$ 1)$

16 A Guide to Automation in Clinical Chemistry May 1969 P. M. G. BROUGHTON $12 \mathrm{~s} 6 \mathrm{~d}(\$ 1.50)$
17 Flame Photometers (2nd edition) 1969 P. WILDING 12s 6d (\$1.50)

18 Control Solutions for Clinical Biochemistry (4th edition) March 1970 P. M. G. BROUGHTON $12 \mathrm{~s} 6 \mathrm{~d}(\$ 1.50)$

19 Spectrophotometers. A comparative list of low-priced instruments readily available in Britain May 1970 c. e. WILDE and P. SEWELL $12 \mathrm{~s} 6 \mathrm{~d}(\$ 1.50)$

20 Quantities and Units in Clinical Biochemistry June 1970 P.M.G. BROUGHTON 12s $6 \mathrm{~d}(\$ 1.50)$ More than 30 copies in units of 10 at 